\title{
Using Data Mining Findings to Aid Searching for Better Cognitive Models
}

\author{
Mingyu Feng ${ }^{1}$, Neil T. Heffernan ${ }^{2}$, and Kenneth Koedinger ${ }^{3}$ \\ ${ }^{1}$ SRI International, Menlo Park, CA 94025 \\ ${ }^{2}$ Worcester Polytechnic Institute, Worcester, MA 01609 \\ ${ }^{3}$ Carnegie Mellon University, Pittsburgh, PA 15213 \\ mingyu.feng@sri.com, nth@wpi.edu, koedinger@cmu.edu
}

\begin{abstract}
One key component of creating an intelligent tutoring system is forming a model that monitors student behavior. Researchers in machine learning area have been using automatic/semi-automatic techniques to search for cognitive models. One of the semi-automatic approaches is learning factor analysis, which involves human making hypothesis and identifying difficulty factors in the related items. In this paper, we propose a hybrid approach in which we leverage findings from our previous educational data mining work to aid the search for a better cognitive model and thus, improve the efficiency of LFA. Preliminary results suggest that our approach can lead to significantly better fitted cognitive models fast.
\end{abstract}

Keywords: Data mining, cognitive model, learning factor analysis.

\section{Introduction}

One key component of creating an intelligent tutoring system (ITS) is forming a model that monitors student behavior. An ITS needs the construction of complex models to represent the skills that students are using and their knowledge states, tracks their progress and chooses what problems will be displayed next. Using a better cognitive model, a system should be able to do a better job of predicting which items students will get correct in real-time and thus do a better job of selecting the next best item. A better model would also help teachers adjust their instruction in a data-driven manner. Given the importance of cognitive models, their construction and improvement has been a major focus in the community. Researchers in machine learning area have been using techniques such as the rule space method [6], Q-matrics [1] learning factor analysis (LFA) [2], learning factor transfer [5] to build/search for cognitive models. We propose a hybrid approach to leverage findings from educational data mining to aid searching for a better cognitive model using LFA.

\section{Methods}

Cen, Koedinger \& Junker [2] proposed a generic, computation intensive method called learning factor analysis (LFA) for cognitive model evaluation and refinement. 
LFA aims to "combine statistics, human expertise and combinatorial search to evaluate and improve a cognitive model". In LFA, a difficulty factor is a hidden feature in a problem that makes the problem easier or harder to solve. An example factor in math with two possible values is using a rule CIRCLE-AREA (e.g. $\mathrm{S}=\pi^{*} \mathrm{r}^{2}$ ) forward (to calculate circle area given radius) or backward (to calculate radius given circle area). Here forward and backward are values of a difficulty factor. Given the difficulty factor, LFA could apply one of the three operators "split", "add", and "merge" on skills in current based model to generate sub-models and perform combinatorial search to look for the "best" model. By applying operator "add" to existing cognitive model, it is hypothesized that there is an unrepresented skill required by the items that are associated with a difficulty factor. The "merge" operator assumes students only need one representation for multiple skills; yet the "split" operator hypothesizes multiple representations be used to represent the variation in one piece of knowledge component. Various heuristics such as Akaike's information criterion (AIC), Bayesian Information Criterion (BIC), R-square and Log likelihood, have been considered as model evaluation and selection measures.

We [3] have conducted a focused item-level analysis of a subset of items to track how student performance on items changed during the same session in a web-based tutoring system (ASSISTments). Items that have same deep features or knowledge requirements, such as approximating square roots, but have different surface features, such as cover stories, were organized into a Group of Learning OPportunity (GLOP). We reported how we could reliably tell which item) is most effective at causing learning. We found out the items vary in their instructional effectiveness of the skill(s) associated with the group of items.

Now that we could reliably tell difference of learning among items, we wanted to employ this information to improve existing cognitive models. As a basis of LFA, the identification of difficulty factors needs human expertise. They have always been found by subject experts through a process of "difficulty factor assessment" [4]. Based upon theory or task analysis, researchers hypothesized the likely factors that cause student difficulties, and by assessing performance difference on pairs of problems that vary by only one factor, the experts identified the hidden knowledge component that could be used to improve a cognitive model. Can we raise efficiency of LFA by suggesting difficulty factors automatically yet still get better models? We have found certain items in a random sequence cause significantly less learning than others. Intuitively, it is highly possible that there is certain factor inherited in the items, which makes it harder for the learning from this item to transfer to later items. This could be either because later items demand more skills than the current one, or because what a student learns from a current item does not help later items. In both conditions, there is probably "mis-tagging" with this item. Presumably, such a factor can be utilized by LFA to manipulate the original cognitive model to search for the best-fit model.

In order to test this idea, we create factor tables for each of the GLOPs. In each table, we use one factor with two values "High" and "Low" indicating the effectiveness of the items. The item that has caused least learning is associated with the factor value "Low" while all other items are associated with "High". Given the factor tables, we ran LFA search over all the GLOPs. BIC was used as the heuristic to evaluate the models in that it balances simplicity and predictive power of models. Among the 38 GLOPs, LFA was able to find statistically significantly better models (a difference of 10 points or more on BIC) for 12 of them, using the factors as assigned in the factor tables. Among the 12 GLOPs, 5 of them included 2 items; 3 included 4 items; the rest 
4 GLOPs had 5, 6, 8, and 9 items respectively. For 11 out of the 12 GLOPs, the application of the "add" operator leaded to a better fitted model, which suggests that there were more knowledge other than the current skills that needed to be represented in the cognitive model in order to better track student learning. We have also conducted a sanity check where we randomly assign one item each GLOP with the "Low" value of the factor, and then run the same searching process. Obviously, for the 2-item GLOPs, the results will be the same as before. But for GLOPs with more items, the search process using randomly assigned factor values only find better models for 2 out of the 27 GLOPs, which makes our previous results of 7 out of 27 somewhat impressive. The results show some validity for the very simple way of suggesting factors.

\section{Conclusion}

This paper describes one practice on how to use educational data mining findings to help improve cognitive modeling. A semi-automatic approach, LFA, is considered. Preliminary results show findings in item effectiveness can be used to assign difficulty factors for items, and thus, automate LFA so that it can efficiently search for superior cognitive models. In terms of future work, we would like to apply this approach on data collected from other tutoring systems to verify the generality.

\section{Acknowledgements}

We would like to acknowledge funding from the US Department of Education, the National Science Foundation, the Office of Naval Research and the Spencer Foundation. All of the opinions expressed in this paper are those solely of the authors and not those of our funding organizations.

\section{References}

1. Barnes, T.: Q-matrix Method: Mining Student Response Data for Knowledge. In: Beck, J. (ed.) Educational Data Mining: Papers from the 2005 AAAI Workshop. AAAI Press, Menlo Park (2005)

2. Cen, H., Koedinger, K., Junker, B.: Learning factors analysis: a general method for cognitive model evaluation and improvement. In: Ikeda, M., Ashley, K.D., Chan, T.-W. (eds.) ITS 2006. LNCS, vol. 4053. Springer, Heidelberg (2006)

3. Feng, M., Heffernan, N., Beck, J.: Using learning decomposition to analyze instructional effectiveness in the ASSISTment system. In: Dimitrova, Mizoguchi, du Boulay (eds.) Proceedings of the 14th International Conference on Artificial Intelligence in Education (AIED 2009). IOS Press, Amsterdam (2009)

4. Koedinger, K.: Research statement for Dr. Kenneth R. Koedinger (June 2000), http://pact.cs.cmu. edu/koedinger/koedingerReserach.html

5. Pavlik, P.I., Cen, H., Koedinger, K.R.: Learning factors transfer analysis: Using learning curve analysis to automatically generate domain models. In: Barnes, Desmarais, Romero, Ventura (eds.) Proceedings of the $2^{\text {nd }}$ International Conference on Educational Data Mining, Cordoba, Spain, pp. 121-130 (2009)

6. Tatsuoka, K.: Rule space: An approach for dealing with misconceptions based on item response theory. Journal of Education Measurement 20(4), 345-354 (1983) 